\title{
Sex differences in 90-day outcomes after mechanical thrombectomy for acute ischemic stroke
}

\author{
Tracy E Madsen, ${ }^{1}$ Eliza DeCroce-Movson, ${ }^{2}$ Morgan Hemendinger, ${ }^{3}$ \\ Ryan A McTaggart, ${ }^{3,4}$ Shadi Yaghi, ${ }^{3}$ Shawna Cutting, ${ }^{3}$ Karen L Furie, ${ }^{3}$ Ali Saad, ${ }^{3}$ \\ Matthew S Siket, ${ }^{1}$ Mahesh V Jayaraman ${ }^{3,4}$
}

${ }^{1}$ Department of Emergency Medicine, Alpert Medical School of Brown University, Providence, Rhode Island, USA

${ }^{2}$ Alpert Medical School of Brown University, Providence, Rhode Island, USA

${ }^{3}$ Department of Neurology, Alpert Medical School of Brown University, Providence, Rhode Island, USA

${ }^{4}$ Department of Radiology, Alpert Medical School of Brown University, Providence, Rhode Island, USA

\section{Correspondence to}

Dr Tracy E Madsen, Department of Emergency Medicine, Alpert Medical School of Brown University, Providence, RI 02903,USA; Tracy_Madsen@ brown.edu

Received 1 May 2018 Revised 18 July 2018 Accepted 21 July 2018 Published Online First 10 August 2018

\section{ABSTRACT}

Background It is largely unknown whether functional outcomes after mechanical thrombectomy for large vessel occlusion (LVO) ischemic strokes differ by sex in non-clinical trial populations. We investigated sex differences in 90-day outcomes among ischemic stroke patients receiving mechanical thrombectomy.

Methods This was a prospective cohort of adults treated with mechanical thrombectomy for LVO at a single academic comprehensive stroke center from July 2015 to April 2017. Data on independence (mRS $\leq 2)$ at hospital discharge and 90 days were collected prospectively. Multiple logistic regression was used to determine the association between sex and 90-day independence, first adjusting for demographics, prestroke mRS, and NIHSS, then by co-morbidities and time to thrombectomy, and finally by vessel recanalization and use of intravenous thrombolysis.

Results We included 279 patients, $52 \%$ of whom were female. Compared with males, females were older (median years (IQR) 81 (75-88) vs. 71.5 (60-81) $\mathrm{P}<0.001$ ) and had higher baseline NIHSS (mean SD $18.2 \pm 7.5$ vs . $16.0 \pm 7.1, P=0.02$ ). Similar proportions of males and females had pre-stroke mRS $\leq 2(73.3 \%$ vs.67.1\%, $\mathrm{P}=0.27)$. In multivariate analyses, males and females had a similar likelihood of being independent at discharge (aOR $0.71(95 \% \mathrm{Cl} 0.32$ to 1.58$)$ ), but females were less likely to be independent at 90 days (aOR 0.37 $95 \% \mathrm{Cl} 0.16$ to 0.87 ).

Conclusions In patients treated with mechanical thrombectomy for LVOs at a large comprehensive stroke center, females were less likely to be independent at 90 days. Future research should investigate contributors to poor outcomes post-discharge in females with LVOS, along with potential interventions to improve outcomes.

\section{INTRODUCTION}

Mechanical thrombectomy has been shown to improve 90-day functional outcomes and to reduce post-stroke disability among patients with ischemic stroke caused by large vessel occlusions (LVOs), ${ }^{1-5}$ and the utilization of mechanical thrombectomy for acute ischemic stroke has increased following the publication of multiple randomized controlled trials demonstrating its effectiveness. ${ }^{6}$ Despite evidence supporting the overall effectiveness of mechanical thrombectomy for LVO strokes, it is unclear whether outcomes after mechanical thrombectomy for LVOs differ by sex in non-trial populations. Understanding the role of sex in outcomes after mechanical thrombectomy is especially important to consider in light of recent data demonstrating the effectiveness of mechanical thrombectomy in selected patients up to 24 hours' post-stroke, data which will further increase the use and potential impact of this intervention in both females and males. ${ }^{78}$ As thrombectomy is being offered to more patients, identifying sex differences in post-intervention outcomes could lead to the consideration of sex-specific approaches to patient selection or even to the procedure itself.

An analysis of the impact of sex on the efficacy of endovascular therapy using data from the Multicenter Randomized Clinical Trial of Endovascular Treatment for Acute Ischemic Stroke in the Netherlands (MR. CLEAN) trial suggested that females were less likely to benefit from intra-arterial treatment and more likely to die following intra-arterial treatment compared with males. ${ }^{9}$ In contrast, a subsequent meta-analysis of data pooled from all five original clinical trials, the Highly Effective Reperfusion evaluated in Multiple Endovascular Stroke Trials (HERMES) collaboration, showed no differences in treatment effect between females and males.$^{10}$ Despite data from MR. CLEAN and HERMES, it is largely unknown whether functional outcomes after mechanical thrombectomy for LVO strokes in 'real world' populations differ between males and females. One small study of patients undergoing mechanical thrombectomy for LVO stroke showed smaller cerebral vessel diameter in females compared with males, but was underpowered to detect differences in outcomes based on sex or vessel diameter. ${ }^{11}$

Extensive previous literature demonstrates that compared with males, females with ischemic stroke have worse functional outcomes regardless of treatment modality, ${ }^{12-16}$ and there are conflicting data regarding the efficacy of mechanical thrombectomy by sex within clinical trials. The objective of this study was to determine whether there are sex differences in functional outcomes following mechanical thrombectomy in a non-clinical trial population.

\section{METHODS}

\section{Study design and patient population}

We conducted a prospective cohort study of adults ( $\geq 18$ years old) treated with mechanical thrombectomy for LVO ischemic stroke at a single, large, academic comprehensive stroke center in the northeast U.S. between July 1, 2015 and April 24, 2017. 
The start date was chosen at the time of study design based on the implementation date of new site protocols for mechanical thrombectomy selection based on published clinical trials. ${ }^{1-5}$ The end date was chosen based on the availability of 90-day outcome data. All adults who underwent mechanical thrombectomy for LVO ischemic stroke during the study period were included. Criteria for performance of mechanical thrombectomy at the study site during the study period included ischemic stroke caused by a proximal large vessel occlusion (intracranial internal carotid artery, M1/proximal M2 branch of middle cerebral artery, or basilar artery) on CT angiogram and arrival within 16 hours of symptom onset or last known time the patient was normal. Both patients who initially presented to the study hospital as well as those who were transferred from an outside hospital were included. The study was approved by the hospital's institutional review board.

\section{Data collection}

Data on our primary outcome, 90-day modified Rankin scale (mRS), were collected prospectively by trained stroke unit staff who spoke with the patients 90 days after their discharge via telephone. Trained stroke unit staff also collected data on planned secondary outcome variables including: functional independence at time of hospital discharge; vessel recanalization immediately following mechanical thrombectomy as defined by TICI score $\geq 2 b$ (perfusion of at least half of the vascular distribution of the occluded artery); complications following thrombectomy: symptomatic intracranial hemorrhage $(\mathrm{sICH})$, post-treatment hemorrhage on imaging defined by hemorrhagic infarction or parenchymal hemorrhage, deep vein thrombosis, pulmonary embolism, and pneumonia; and hospital readmission within 90 days. These data were entered into the study institution's stroke registry that is maintained using RedCap software. Additional data that were entered into the registry by trained stroke unit staff and/or trained research assistants included patient demographics, medical co-morbidities, pre-stroke mRS, presenting NIHSS, and whether patients were treated with intravenous (IV) tissue plasminogen activator.

\section{Data analysis}

Descriptive statistics (means, medians, proportions as appropriate) were used to compare baseline demographic and clinical characteristics (sex, age, race, marital status, insurance status, NIHSS, location of vessel occlusion, pre-stroke functional status, and comorbidities) between females and males. Next, unadjusted functional outcomes (mRS scores) were compared between females and males at discharge and at 90 days, first as an ordinal variable and then as a binary variable using chi-square tests. Functional independence was defined as $\mathrm{mRS} \leq 2$ compared with $\mathrm{mRS}>2$.

For our primary outcome, independence at 90 days' poststroke $(\mathrm{mRS} \leq 2)$, unadjusted logistic regression was conducted followed by multivariate logistic regression with sex as the primary independent variable. In our first multivariate model, we adjusted for age (standardized to account for a non-parametric distribution), race (white/non-white), marital status (married vs. divorced/single/widowed), insurance status (private/ government-sponsored), independence prior to stroke (baseline $m R S \leq 2 / m R S>2$ ), and initial NIHSS. Race was categorized as a binary variable due to small numbers of participants from individual race categories, but frequencies in individual categories were reported. In the second model, in addition to adjusting for variables listed in the first model, we adjusted for co-morbidities (atrial fibrillation/flutter, hypertension, dyslipidemia, diabetes, coronary artery disease (CAD)/myocardialinfarction (MI)) and time from symptom onset or last known normal to groin puncture for mechanical thrombectomy (standardized to account for a non-parametric distribution). Finally, we adjusted for all variables in the first two models with the addition of treatment with IV tPA and recanalization as defined by TICI $2 \mathrm{~b}$ or greater. A pre-specified sensitivity analysis was performed with vessel recanalization defined by TICI 3 or greater. Odds ratios (ORs) with 95\% confidence intervals (CIs) were reported for regression models. Models were tested for fit using Hosmer-Lemeshow tests and were also evaluated for collinearity.

Some post-hoc sensitivity and/or subgroup analyses were performed as well. First, location of vessel occlusion (ICA/M1/ $\mathrm{M} 2 /$ basilar) was added to the multivariate model for outcomes at 90 days. We also performed an analysis using the time to recanalization variable rather than time to groin puncture to account for potential differences in procedural difficulty: this was not chosen for the main analysis, though, because the time to recanalization was similar by sex, and not all patients recanalized. Finally, post-hoc subgroup analysis by age $(<75$ years and $\geq 75$ years old) was performed given the significant age differences in our patient sample by sex.

All secondary outcomes were specified a priori but were considered to be hypothesis-generating. To compare functional independence at hospital discharge, multivariate logistic regression was used to compare outcomes by sex, adjusting for the same covariates used in the 90-day outcome models. Our second group of secondary outcomes were time metrics relevant to mechanical thrombectomy. Specifically, females and males were compared with respect to the following thrombectomy-related metrics using Wilcoxon rank sum tests: time from onset of symptoms to arrival at first hospital; time from arrival at first hospital to start of thrombectomy; and time from arrival at first hospital to vessel recanalization. For additional secondary outcomes (readmission to the hospital within 90 days and in-hospital complications (sICH, post-stroke hemorrhage, deep venous thrombosis, pulmonary embolus, and pneumonia)), chi-square tests were used to compare females and males, and frequencies and proportions were reported.

For all analyses, P-values less than 0.05 were considered to be significant. Stata version 13.0 was used for all analyses. ${ }^{17}$

\section{RESULTS}

Two-hundred and seventy-nine patients were treated with mechanical thrombectomy during the study period. Of 279, $52 \%$ were females, $14 \%$ were non-white, and $4.3 \%$ were Hispanic. Further breakdown by race can be found in table 1 . Compared with males, females were 10 years older (median years: 81 (IQR 75-88) vs. 71.5 (IQR 60-81), P<0.001), had higher initial NIHSS scores (mean (SD): 18.2 (7.5) vs. 16.0 (7.1), $\mathrm{P}=0.02$ ), were less likely to be married (38.2\% vs. $67.5 \%$, $\mathrm{n}=0.004)$, and were more likely to have government-sponsored insurance $(86.8 \%$ vs. $73.1 \%, \mathrm{P}=0.004)$. The location of vessel occlusion was similar by sex (table 1 ). The majority of patients had anterior circulation strokes: $8.3 \%$ of females vs. $7.6 \%$ of males had basilar strokes. Otherwise, baseline characteristics including medical history and percentage of patients that were independent prior to stroke were similar between males and females (table 1).

With regard to our primary outcome, independence at 90 days' post-stroke, data on 90-day mRS were missing on 24 participants, leaving 255 participants for this analysis. Of $255,27 \%$ of females $(n=37)$ vs,.46.6\% of males $(n=55)$ were independent at 90 -days' post-stroke $(\mathrm{P}=0.001)$. Unadjusted, females were $58 \%$ less likely 
Table 1 Baseline characteristics of patients treated with mechanical thrombectomy for large vessel occlusion ischemic stroke by sex

\begin{tabular}{|c|c|c|c|}
\hline & $\begin{array}{l}\text { Females } \\
\mathrm{N}(\%)^{*}\end{array}$ & $\begin{array}{l}\text { Males } \\
\mathrm{N}(\%)^{*}\end{array}$ & P-value \\
\hline Age (median (IQR)) & $81(75-88)$ & $71.5(60-81)$ & $<0.001$ \\
\hline Race & & & 0.14 \\
\hline White & $117(80.7)$ & $106(79.1)$ & \\
\hline Black & $6(4.1)$ & $8(6.0)$ & \\
\hline Asian & $1(0.7)$ & 0 & \\
\hline Other & $14(9.7)$ & $6(4.5)$ & \\
\hline Unknown & $7(4.8)$ & $14(10.4)$ & \\
\hline Ethnicity & & & 0.43 \\
\hline Non-Hispanic & $127(87.6)$ & $117(87.3)$ & \\
\hline Hispanic & $8(5.5)$ & $4(3.0)$ & \\
\hline Unknown & $10(6.9)$ & $13(9.7)$ & \\
\hline Baseline NIHSS (mean (SD)) & $18.2(7.5)$ & $16.0(7.1)$ & 0.02 \\
\hline Anatomic location & & & 0.79 \\
\hline ICA & $20(13.8)$ & $24(18.3)$ & \\
\hline M1 & $90(62.9)$ & $80(61.0)$ & \\
\hline M2 & $21(14.7)$ & $17(12.9)$ & \\
\hline Basilart & $12(8.4)$ & $10(7.6)$ & \\
\hline $\begin{array}{l}\text { Independence prior to stroke (pre- } \\
\text { stroke } m R S \leq 2 \text { ) }\end{array}$ & $96(73.3)$ & $96(67.1)$ & 0.27 \\
\hline Married & $52(38.2)$ & $85(67.5)$ & $<0.001$ \\
\hline Insurance status & & & 0.004 \\
\hline Private & $19(13.2)$ & $36(26.9)$ & \\
\hline $\begin{array}{l}\text { Government-sponsored } \\
\text { (Medicare/Medicaid) }\end{array}$ & $125(86.8)$ & $98(73.1)$ & \\
\hline History of TIA or stroke & $32(22.2)$ & $33(24.6)$ & 0.636 \\
\hline History of CAD or MI & $33(24.6)$ & $36(30.5)$ & 0.296 \\
\hline History of DM & $23(15.9)$ & $31(23.1)$ & 0.202 \\
\hline History of atrial fibrillation & $77(53.5)$ & $65(48.5)$ & 0.422 \\
\hline History of hypertension & $105(72.4)$ & $96(71.6)$ & 0.886 \\
\hline History of hyperlipidemia & $67(46.2)$ & $61(45.5)$ & 0.620 \\
\hline History of smoking & $21(18.4)$ & $37(33.0)$ & 0.012 \\
\hline Current alcohol use & $29(27.6)$ & 47 (49.5) & 0.001 \\
\hline
\end{tabular}

*Data in columns represented as frequencies and proportions unless otherwise specified.

tTwo of these patients had basilar occlusion on imaging but other occlusions on angiogram (one had posterior cerebral artery occlusion, one had superior cerebellar artery occlusion).

NIHSS: National Institute of Health Stroke scale; mRS: modified Rankin scale; TIA: transient ischemic attack; CAD: coronary artery disease; MI: myocardial infarction; DM: diabetes mellitus.

than males to be independent at 90 days' post-stroke $(95 \% \mathrm{CI} 0.25$ to $0.71, \mathrm{P}=0.001$ ). Figure 1 displays the proportion of patients at each mRS level at baseline, discharge, and 90days among females and males, and shows that the distribution of mRS scores post-stroke was less favorable in females than males.

Table 2 shows adjusted estimates of the association between female sex and independent functional status at 90 days' poststroke. At 90 days' post-stroke, controlling for demographics, stroke severity, and pre-stroke functional status reduced the association between sex and functional outcome seen in unadjusted analyses (table 2). Further adjustment for time to thrombectomy and comorbidities in Model 2 and for treatment with IV tPA and vessel recanalization in Model 3, however, increased the effect size and showed that females were less likely than males to be independent even after adjusting for all covariates $(0.37,95 \% \mathrm{CI}$ 0.16 to $0.87, \mathrm{P}=0.02$ ). Model fit was tested using the HosmerLemeshow test: P-values for all models were $>0.05$ indicating adequate fit.

We conducted several sensitivity analyses. First, we conducted a sensitivity analysis with recanalization defined using a cut-off of TICI 3 , and the results were similar: adjusting for all covariates in Model 3, females were less likely to be functionally independent at 90 days (aOR $0.3195 \% \mathrm{CI} 0.13$ to $0.74, \mathrm{P}=0.01$ ). Next, a model that included location of occlusion (M1, M2, or basilar with ICA as the reference group) along with all other covariates in Model 3 resulted in a similar difference in outcomes by sex: females were 0.33 times as likely to be independent at 90 days $(95 \% \mathrm{CI} 0.14$ to $0.79, \mathrm{P}=0.01$ ).

Time to recanalization was also considered. Median time of onset to recanalization was similar between females and males (235 min (IQR 171-354) vs. 243 min (IQR 183-330), respectively). A model using time from stroke onset to recanalization (rather than time to groin puncture) was used, again adjusting for all covariates in Model 3, and odds of independence at 90 days was 0.35 (95\%CI 0.15 to $0.83, \mathrm{P}=0.02)$.

When multivariate logistic regression was performed in subgroups by age, among those less than 75 years' old, the OR for independence for females vs. males was 0.24 (95\% CI 0.05 to $1.09, \mathrm{P}=0.06)$. Among those 75 years of age or greater, $\mathrm{OR}$ for females vs. males was 0.54 (95\% CI 0.12 to $2.32, \mathrm{P}=0.41)$.

With regard to functional outcomes at the time of hospital discharge, there was a trend toward females being less likely to be functionally independent compared with males in unadjusted analysis (aOR 0.60 (95\%CI 0.35 to 1.02$)$; $\mathrm{P}=0.06$ ), but after adjusting for demographics, pre-stroke functional status, co-morbidities, time to thrombectomy, vessel recanalization, and treatment with IV tPA, the association between sex and functional independence was not significant (aOR 0.71 (95\% CI 0.32 to 1.58$) \mathrm{P}=0.41$ ).

Table 3 shows relevant time metrics by sex. In brief, females and males had similar times between symptom onset and hospital arrival, between hospital arrival and start of thrombectomy, and between hospital arrival and recanalization.

For additional secondary outcomes, in-hospital complications were similar between females and males, respectively (sICH: $4.2 \%(n=6)$ vs. $6.1 \%(n=8), P=0.47$; deep venous thrombosis: $0.7 \%(n=1)$ vs. $0.7 \%(n=1), P=0.95$; pulmonary embolus: $2.1 \%$ $(\mathrm{n}=3)$ vs. $0.7 \%(\mathrm{n}=1), \mathrm{P}=0.35$; pneumonia: $9 \%(\mathrm{n}=13)$ vs. $8.9 \%(\mathrm{n}=12), \mathrm{P}=0.99)$. Post-treatment hemorrhage on imaging was similar by sex: $87.3 \%$ of males vs. $86.6 \%$ of females had no hemorrhage, and $12.7 \%$ of males had hemorrhagic infarctions or parenchymal hematomas compared with $13.3 \%$ of females $(\mathrm{P}=0.72)$. Hospital readmissions within 90 days were also similar by sex $(36.7 \%(\mathrm{n}=25)$ of females vs. $33.8 \%$ of males $(\mathrm{n}=24), \mathrm{P}=0.71)$.

\section{DISCUSSION}

In summary, in our study of patients with LVO ischemic strokes treated with mechanical thrombectomy, females were less likely to be independent at 90 days' post-stroke and overall had worse functional outcomes as measured by the modified Rankin scale 90 days' post-stroke, even after adjusting for factors including age, stroke severity, and baseline functional status. Our study findings are consistent with previous studies showing sex differences in outcomes among populations of ischemic stroke patients, ${ }^{12-16}$ but also demonstrate that these differences in outcome persist among 
those treated with mechanical thrombectomy, a therapy known to be highly effective for the treatment of LVO strokes. ${ }^{1-5}$ Our findings also add to the literature by demonstrating sex differences in outcomes after mechanical thrombectomy in a non-clinical trial population and are thus potentially generalizable to other populations of patients with LVOs treated with thrombectomy at other geographic locations and institutions. Of note, one previous study of sex differences in outcomes after thrombectomy suggests that smaller intracerebral vessel diameter could be a contributor to such differences. ${ }^{11}$ In this particular study, both the ICA and MCA were smaller in females than males, but neither sex nor vessel diameter were significant predictors of outcome in multivariate analyses. ${ }^{11}$ The study, however, was underpowered to detect outcome differences by sex or vessel diameter. ${ }^{11}$

The difference in functional status in females compared with males was most pronounced at the 90-day mark, more so than at the time of hospital discharge. This finding, combined with data showing similar rates of vessel recanalization between females and males, a known predictor of favorable outcomes, ${ }^{18} 19$ suggest that the worse outcomes in females are not likely related to the thrombectomy itself but are a result of other unmeasured contributors that affect recovery post-stroke and after hospital discharge. Our data showing similar in-hospital complication

\section{Women}

6.3 15.4 26.6

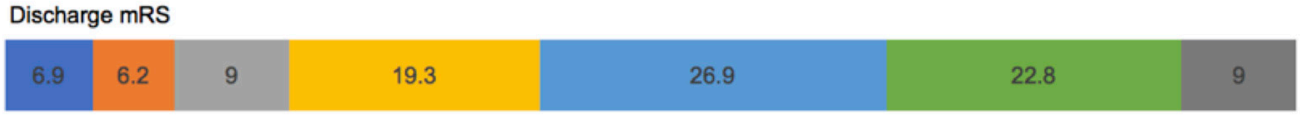

90-day $\mathrm{mRS}$

Pre-stroke mRS

Men

\section{8}

rates by sex support this finding. Unmeasured factors that may contribute to worse outcomes in females at the 90-day mark include frailty, ${ }^{20}$ musculoskeletal co-morbidities such as arthritis and osteoporosis that are more common in females than males, ${ }^{21}$ and mental health disorders, ${ }^{21}$ all of which have potential interventions. Previous data show that females also have a worse quality of life $^{22} 23$ and more post-stroke depression compared with $\mathrm{m}^{\text {ales:24 } 25}$ these factors likely contribute to more activity limitations as measured by modified Rankin scales but were not accounted for in our study.

Consistent with prior studies, we found that favorable baseline functional status, or being independent prior to stroke, was a predictor of the independence at 90 days' post-thrombectomy. ${ }^{1426}$ While this finding is not surprising, it is notable that controlling for baseline functional status, age, and other relevant variables did not eliminate the association between patient sex and functional outcomes. These findings suggest that baseline functional status and age do not adequately capture the clinical, social, and/or cultural factors that may affect outcomes in females post-stroke. For example, social isolation is a factor which has been shown to be associated with poor outcomes post-stroke ${ }^{27}$ but was not available for inclusion in our study. We did control for marital status, but this variable likely does not adequately 
Table 2 Multivariate analyses of association between sex and independence at 90 days among patients that received mechanical thrombectomy for large vessel occlusion ischemic stroke

\begin{tabular}{|c|c|c|}
\hline & 90-day mRS & $P$ value \\
\hline Unadjusted & $0.42(0.25-0.71)$ & 0.001 \\
\hline $\begin{array}{l}\text { Model 1: Adjusted for demographics, pre-stroke } \\
\text { functional status, stroke severity* }\end{array}$ & $0.50(0.25-1.00)$ & 0.05 \\
\hline $\begin{array}{l}\text { Model 2: Adjusted for model } 1+\text { risk factors/ } \\
\text { comorbidities+time from symptom onset to } \\
\text { thrombectomy } \dagger\end{array}$ & $0.37(0.16-0.85)$ & 0.02 \\
\hline $\begin{array}{l}\text { Model 3: Adjusted for model 2+TICl score (2B or } \\
\text { more)+IV tPA }\end{array}$ & $0.37(0.16-0.87)$ & 0.02 \\
\hline \multicolumn{3}{|c|}{$\begin{array}{l}\text { *Model } 1 \text { included sex, age (standardized), race, marital status, pre-stroke } \\
\text { functional status, and NIHSS. } \\
\text { †Model } 2 \text { included all variables in Model } 1 \text { with addition of co-morbidities (atrial } \\
\text { fibrillation, hyperlipidemia, hypertension, diabetes, coronary artery disease/ } \\
\text { myocardial infarction) and time from symptom onset to start of thrombectomy } \\
\text { (standardized). } \\
\text { łModel } 3 \text { included all variables in Model t2 with addition of treatment with } \\
\text { intravenous tPA and vessel recanalization as defined by TICI } 2 \text { b or better. For } \\
\text { standardized variables, aOR represents the change in odds of having independent } \\
\text { functional status at } 90 \text { days' post-stroke for every one SD increase in the variable } \\
\text { of interest. } \\
\text { mRS, modified Rankin scale. }\end{array}$} \\
\hline
\end{tabular}

capture the effects of social isolation on health outcomes. It is also likely that there are sex differences in the cellular mechanisms of brain recovery post-stroke which contribute to sex differences in clinical outcomes, a hypothesis which is supported by some pre-clinical data ${ }^{28}$ but would require further research and confirmation.

Our post-hoc analysis of sex differences in functional independence in subgroups by age suggests that sex differences may be greater among those under 75 years compared with those older than 75 years, but these data should be interpreted with caution as the analyses are likely underpowered, and results may differ based on the age cut-off chosen. Future research could attempt to power analyses to be able to look at sex differences in younger and older patients separately.

Our study had several limitations. As discussed, we were not able to evaluate the effect of several factors which may disproportionately affect long-term post-stroke outcomes in females including frailty, musculoskeletal co-morbidities, psychiatric disorders, cognitive impairment, and social factors such as living arrangement and social isolation. We also did not have data on vessel diameter or prevalence of intracranial atherosclerotic disease. In addition, our study was not designed to investigate the effectiveness of thrombectomy compared with alternative medical treatments. Because all patients included in the study had mechanical thrombectomy performed, we cannot comment

Table 3 Time metrics for mechanical thrombectomy by sex

\begin{tabular}{llll}
\hline & Females & Males & P value \\
\hline $\begin{array}{l}\text { Symptom onset to arrival at } \\
\text { hospital* (minutes) }\end{array}$ & $63.5(39-168)$ & $60(35-146)$ & 0.48 \\
$\begin{array}{l}\text { Hospital arrival* to start of } \\
\text { thrombectomy procedure } \\
\text { (minutes) }\end{array}$ & $116(82-154)$ & $117.5(92.5-152)$ & 0.55 \\
$\begin{array}{l}\text { Hospital arrival }{ }^{*} \text { to vessel } \\
\text { recanalization (minutes) }\end{array}$ & $141(106-195)$ & $151.5(123-183.5)$ & 0.43 \\
\hline
\end{tabular}

*All metrics pertain to arrival at first hospital in those patients who were transferred to the study site from another institution. All values displayed are medians with interquartile ranges. on the efficacy of mechanical thrombectomy in females vs. males. Finally, we do not have data on the cause of death after hospital discharge; such data could provide additional insight into sex differences in 90-day outcomes and potential interventions.

\section{CONCLUSIONS}

In conclusion, in our study of patients undergoing thrombectomy for LVO ischemic stroke, females had worse functional outcomes at 90 days, even after accounting for age, pre-stroke functional status, stroke severity, and medical co-morbidities. Our data suggest that sex differences in outcomes are also not explained by vessel recanalization or in-hospital complications. Future research should continue to investigate contributors to sex differences in outcomes among patients with large vessel occlusions treated with mechanical thrombectomy. Such research could help to identify modifiable factors and interventions that could maximize long-term post-stroke outcomes and thus the public health impact of acute interventions.

Contributors TM is responsible for study conception, design, data acquisition, analysis, interpretation as well as drafting the manuscript and critically revising it. $\mathrm{ED}$ and $\mathrm{MH}$ contributed to data acquisition and critical revisions of the manuscript. RM, SY, and MJ contributed to study design, data acquisition and interpretation, and critical revisions of the manuscript. SC, KF, AS, and MS contributed to data interpretation and critical revisions of the manuscript. All authors have approved the final manuscript and are accountable for all aspects of the work.

Funding The authors have not declared a specific grant for this research from any funding agency in the public, commercial, or not-for-profit sectors.

Competing interests $\mathrm{MJ}$ is a consultant for Medtronic (minor) and a consultant for Stream Biomedical (minor).

Patient consent Not required.

Ethics approval Rhode Island Hospital Institutional Review Board.

Provenance and peer review Not commissioned; externally peer reviewed.

Data sharing statement The data is available upon request from the corresponding author.

\section{REFERENCES}

1 Berkhemer OA, Fransen PS, Beumer D, et al. A randomized trial of intraarterial treatment for acute ischemic stroke. N Engl J Med 2015;372:11-20.

2 Campbell BC, Mitchell PJ. EXTEND-IA Investigators. Endovascular therapy for ischemic stroke. N Engl J Med 2015;372:2365-6.

3 Goyal M, Demchuk AM, Menon BK, et al. Randomized assessment of rapid endovascular treatment of ischemic stroke. N Engl J Med 2015;372:1019-30.

4 Jovin TG, Chamorro A, Cobo E, et al. Thrombectomy within 8 hours after symptom onset in ischemic stroke. N Engl J Med 2015:372:2296-306.

5 Saver JL, Goyal M, Bonafe A, et al. Stent-retriever thrombectomy after intravenous t-PA vs. t-PA alone in stroke. N Eng/ J Med 2015;372:2285-95.

6 Hussein HM, Saleem MA, Qureshi Al. Statewide Trends in Utilization and Outcomes of Endovascular Treatment of Acute Ischemic Stroke: Analysis of Minnesota Hospital Association Data (2014 and 2015). J Stroke Cerebrovasc Dis 2018:27:677-81.

7 Albers GW, Marks MP, Kemp S, et al. Thrombectomy for stroke at 6 to 16 hours with selection by perfusion imaging. N Eng/ J Med 2018:378:708-18.

8 Nogueira RG, Jadhav AP, Haussen DC, et al. Thrombectomy 6 to 24 hours after stroke with a mismatch between deficit and infarct. N Eng/ J Med 2018;378:11-21.

9 de Ridder IR, Fransen PS, Beumer D, et al. Is Intra-arterial treatment for acute ischemic stroke less effective in women than in men? Interv Neurol 2016:5(3-4):174-8.

10 Goyal M, Menon BK, van Zwam WH, et al. Endovascular thrombectomy after large-vessel ischaemic stroke: a meta-analysis of individual patient data from five randomised trials. Lancet 2016:387:1723-31.

11 Davison MA, Ouyang B, Keppetipola KM, et al. Arterial diameter and the gender disparity in stroke thrombectomy outcomes. J Neurointerv Surg 2018. doi: 10.1136/ neurintsurg-2017-013697. [Epub ahead of print 12 Feb 2018].

12 Denti L, Artoni A, Scoditti U, et al. Impact of gender-age interaction on the outcome of ischemic stroke in an Italian cohort of patients treated according to a standardized clinical pathway. Eur J Intern Med 2013;24:807-12.

13 Fukuda M, Kanda T, Kamide N, et al. Gender differences in long-term functional outcome after first-ever ischemic stroke. Intern Med 2009:48:967-73.

14 Lisabeth LD, Reeves MJ, Baek J, et al. Factors influencing sex differences in poststroke functional outcome. Stroke 2015;46:860-3. 
15 Maeda K, Toyoda K, Minematsu K, et al. Effects of sex difference on clinical features of acute ischemic stroke in Japan. J Stroke Cerebrovasc Dis 2013;22:1070-5.

16 Silva GS, Lima FO, Camargo EC, et al. Gender differences in outcomes after ischemic stroke: role of ischemic lesion volume and intracranial large-artery occlusion. Cerebrovasc Dis 2010;30:470-5.

17 Stata statistical software: release 13. College Station, TX: StataCorp LP, 2013.

18 Camerlingo M, Tudose V, Tognozzi M, et al. Predictors of re-canalisation in acute cerebral infarction from occlusion of the terminal internal carotid artery or of the middle cerebral artery mainstem treated with thrombolysis. Int J Neurosci 2014;124:199-203.

19 Rha JH, Saver JL. The impact of recanalization on ischemic stroke outcome: a metaanalysis. Stroke 2007:38:967-73.

20 Gordon EH, Peel NM, Samanta M, et al. Sex differences in frailty: a systematic review and meta-analysis. Exp Gerontol 2017;89:30-40.

21 GBD 2016 Disease and Injury Incidence and Prevalence Collaborators. Global, regional, and national incidence, prevalence, and years lived with disability for 328 diseases and injuries for 195 countries, 1990-2016: a systematic analysis for the Global Burden of Disease Study 2016. Lancet 2017;390:1211-59.
22 Bushnell $C D$, Reeves MJ, Zhao $X$, et al. Sex differences in quality of life after ischemic stroke. Neurology 2014;82:922-31.

23 Gargano JW, Reeves MJ. Sex differences in stroke recovery and stroke-specific quality of life: results from a statewide stroke registry. Stroke 2007:38:2541-8.

24 Chen YK, Qu JF, Xiao WM, et al. Intracranial atherosclerosis and poststroke depression in Chinese patients with ischemic stroke. J Stroke Cerebrovasc Dis 2016:25:998-1004.

25 Tsai CS, Wu CL, Hung TH, et al. Incidence and risk factors of poststroke depression in patients with acute ischemic stroke: a 1-year prospective study in Taiwan. Biomed J 2016;39:195-200

26 Renoux C, Coulombe J, Li L, et al. Confounding by pre-morbid functional status in sudies of apparent sex differences in severity and outcome of stroke. Stroke 2017:48:2731-8.

27 Boden-Albala B, Litwak E, Elkind MS, et al. Social isolation and outcomes post stroke. Neurology 2005;64:1888-92.

28 Chauhan A, Moser H, McCullough LD. Sex differences in ischaemic stroke: potential cellular mechanisms. Clin Sci 2017;131:533-52. 